\title{
ESTUDOS COMPARATIVOS DOS MÉTODOS DE CÁLCULO COM RESULTADO EXPERIMENTAL DO ALARGAMENTO NO PROCESSO DE LAMINAÇÃO A QUENTE DO AÇO AISI1020*
}

\author{
Matheus Fortes Stiborski ${ }^{1}$ \\ Luana De Lucca de Costa ${ }^{2}$ \\ André Rosiak \\ Lucas Antônio Gonçalves ${ }^{3}$ \\ Kíssia Carolina Bertoluci Cardoso ${ }^{3}$ \\ Rodrigo Prestes Limberger ${ }^{4}$ \\ Lírio Schaeffer
}

\section{Resumo}

Este estudo descreve a aplicação de três métodos de cálculos teóricos pelos modelos matemáticos de Tafel e Sedlaczek, Siebel e Köster, com o objetivo de calcular o alargamento na laminação a quente de barras em aço AISI 1020 em um laminador tipo duo e definir quais dos modelos de alargamento se aproxima mais dos resultados obtidos experimentalmente. Foram utilizadas amostras com dimensões iniciais de largura $\left(b_{\circ}\right), 37,7 \mathrm{~mm}$, altura $\left(h_{\circ}\right), 15,7 \mathrm{~mm}$, e comprimento $\left(\mathrm{l}_{\circ}\right)$, $300,00 \mathrm{~mm}$ onde se ajustou a luz dos cilindros para que fossem obtidas reduções aproximadas de $15 \%$ e $20 \%$ em altura. Os resultados obtidos indicam que o modelo de Siebel apresenta melhores resultados comparado com os valores experimentais.

Palavras-chave: Laminação a quente; Modelos matemáticos.

\section{COMPARISON OF EXPANSIONBETWEEN MEASURED EXPERIMENTALLY AND MATHEMATICAL MODELS FOR AISI 1020 HOT ROLLING PROCESS \\ Abstract}

This study describes the application of three mathematical models by TafelSedlaczek, Siebel and Köster models to find the expansion in the hot rolling AISI 1020 steel bars in a Duo rolling mill and explain which of these models is closer to the experimental results. The samples with initial dimensions of a width $\left(b_{o}\right), 37.7$ $\mathrm{mm}$, height $\left(h_{0}\right), 15.7 \mathrm{~mm}$, and length $\left(l_{0}\right), 300.00 \mathrm{~mm}$ have been compressed approximately to $15 \%$ and $20 \%$ of initial length by adjusting the cylinder opening. The results indicate that the Siebel model performs approximations closed to the experimental result.

Keywords: Hot rolling; Mathematical models.

1 Graduando em Engenharia Metalúrgica, Laboratório de Transformação Mecânica, Universidade Federal do Rio Grande do Sul (UFRGS), Porto Alegre, Rio Grande do Sul, Brasil.

2 Engenharia Mecânica, Mestre em Engenharia, Doutoranda do Programa de Pós-Graduação em Engenharia de Minas, Metalurgia e Materiais (PPGE3M), UFRGS, Porto Alegre, RS, Brasil.

3 Graduando em Engenharia Metalúrgica, Laboratório de Transformação Mecânica, UFRGS, Porto Alegre, RS, Brasil.

4 Engenheiro Mecânico, Mestrando do PPGE3M, UFRGS, Porto Alegre, RS, Brasil.

5 Dr.Ing., Laboratório de Transformação Mecânica, UFRGS, Porto Alegre, RS, Brasil. 


\section{INTRODUÇÃO}

A laminação é um processo de conformação mecânica que consiste na passagem de um corpo sólido por cilindros metálicos que giram em sentidos opostos com a mesma velocidade que tem como objetivo a redução secção transversal de blocos ou barras, ocasionando aumento no comprimento e melhoria nas propriedades dos materiais [1,2]. A Figura 1 mostra as principais variáveis no processo de laminação.

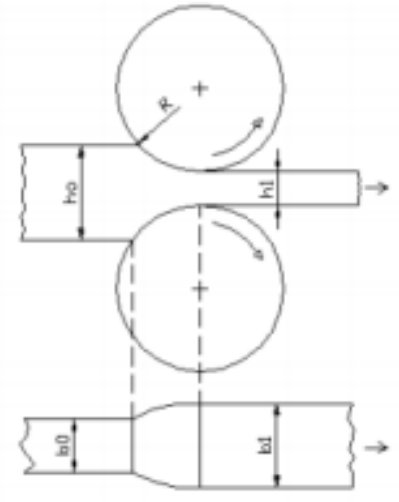

Figura 1 - llustração das principais variáveis do processo de laminação. Onde $\mathrm{R}$ é raio do cilindro, $\mathrm{v}_{\mathrm{o}}$ é a velocidade de saída da barra, $h_{0}$ é altura inicial da barra e $h_{1}$ é a altura final da barra [2].

Na laminação o material é submetido a esforços compressivos resultantes da ação de prensagem dos rolos e a esforços cisalhantes superficiais resultantes do atrito entre os rolos e o material laminado.

$\mathrm{Na}$ laminação a quente é realizada a redução inicial dos lingotes em blocos, tarugos ou placas e, posteriormente, é realizada uma nova etapa de laminação a quente para transformar o produto em chapas grossas, tiras, vergalhões, barras, tubos, trilhos ou perfis estruturais. A laminação a frio que ocorre após a laminação de tiras a quente e produz tiras de excelente acabamento superficial, com boas propriedades mecânicas e controle dimensional do produto final bastante rigoroso [3].

A laminação é utilizada tanto para a produção de produtos planos, como, chapas finas e grossas; e na fabricação de produtos não-planos, como, vergalhões, tubos e perfis estruturais. Os produtos planos são laminados com cilindros lisos enquanto os não-planos em cilindros com canais, conforme Figura 2. A laminação não-plana é mais complexa e é caracterizada por mais parâmetros geométricos [4].

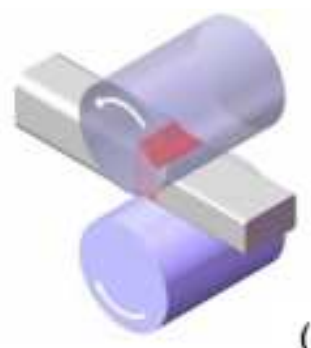

(a)

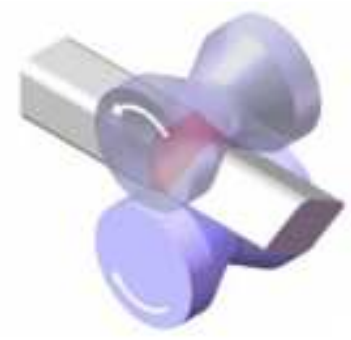

(b)

Figura 2 - Laminação plana (a) e laminação em canais (b) [4].

\subsection{Deformações Ocorridas no Processo de Laminação}

A deformação na laminação pode ser expressa em deformação relativa, deformação absoluta e deformação verdadeira em altura, largura e comprimento, expressas nas equações abaixo [2,4]. 


\begin{tabular}{|c|c|c|c|}
\cline { 2 - 4 } \multicolumn{1}{c|}{} & \multicolumn{1}{c|}{$\begin{array}{c}\text { Deformação } \\
\text { absoluta }\end{array}$} & $\begin{array}{c}\text { Deformação } \\
\text { relativa }\end{array}$ & $\begin{array}{c}\text { Deformação } \\
\text { verdadeira }\end{array}$ \\
\hline Altura & $\Delta h_{i}=h_{i}-h_{i-1}(1)$ & $\varepsilon_{h i}=\frac{\Delta h_{i}}{h_{i-1}}(4)$ & $\varphi_{h i}=\ln \left(\frac{h_{i}}{h_{i-1}}\right)(7)$ \\
\hline Largura & $\Delta b_{i}=b_{i}-b_{i-1}(2)$ & $\varepsilon_{b i}=\frac{\Delta b_{i}}{b_{i-1}}(5)$ & $\varphi_{b i}=\ln \left(\frac{b_{i}}{b_{i-1}}\right)(8)$ \\
\hline Comprimento & $\Delta l_{i}=l_{i}-l_{i-1}(3)$ & $\varepsilon_{l i}=\frac{\Delta l_{i}}{l_{i-1}}(6)$ & $\varphi_{l i}=\ln \left(\frac{L_{i}}{l_{i-1}}\right)(9)$ \\
\hline
\end{tabular}

Onde, em uma barra retangular com área de secção transversal inicial, $A_{i-1}$, altura inicial, $h_{i-1}$, largura inicial, $b_{i-1}$, comprimento inicial, $\mathrm{l}_{\mathrm{i}-1}$, é laminada no passe $\mathrm{i}$, alterando sua área de secção transversal final, $A_{i}$, altura final, $h_{i}$, largura final, $b_{i}$, e comprimento, $I_{i}[4]$.

O volume na laminação não sofre variação, obedecendo a lei da constância de volume. Entretanto, ao passar pelo laminador, a barra sofre redução na direção da altura, aumento sua largura e seu comprimento.

\subsection{Estimativa do Coeficiente de Atrito}

A fórmula de Ekelund, para determinação do coeficiente de atrito, varia com a qualidade do material do cilindro e com a temperatura de laminação ( $\left.T_{\text {lam }}\right)$, onde para cilindros de aço:

$$
\mu=1,05-0,005 \cdot T_{\text {lam }}
$$

\subsection{Condição De Agarre}

Para que ocorra o agarre na barra, é necessário que o ângulo de contato seja menor que o coeficiente de atrito. Como o ângulo de contato aumenta à medida a seção da barra sofre redução, é preciso aumentar o atrito ou buscar forças auxiliares para assegurar a condição de agarre no inicio da laminação [3] a Figura 3 apresenta o esquema simplificado das forças na laminação.

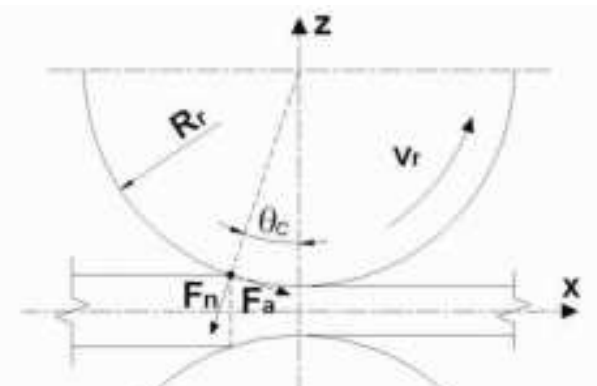

Figura 3 - Esquema simplificado das forças que atuam no plano de entrada da zona de deformação [4].

\subsection{Modelos para o Cálculo de Alargamento}

Os modelos teóricos para estimar o alargamento a cada passe são ferramentas indispensáveis para a previsão do comportamento do material ao passar pelo laminador. 


\subsubsection{Modelo de Siebel}

O modelo de Siebel propõe que o alargamento é diretamente proporcional ao comprimento da projeção do arco de contato.

$$
\Delta b=C_{s} \cdot l d \cdot \frac{\Delta h}{h_{o}}
$$

Onde $\Delta \mathrm{b}$ é a variação absoluta em altura, ld é o arco de contato, $\mathrm{C}_{\mathrm{s}}$ é o coeficiente de correlação da fórmula de Siebel para temperatura de laminação $(0,35$ para aço de construção à $1000^{\circ} \mathrm{C}$ ), $\Delta$ h é a variação da altura e ho é a altura antes da entrada no cilindro.

\subsubsection{Modelo de Tafel e Sedlaczek}

Tafel e Sedlazeck propuseram uma equação para a laminação de perfis planos, em sua forma ampliada [1]:

$$
\Delta b=\frac{0,435 \cdot b_{o} \cdot \Delta h \cdot \sqrt{b_{o} \cdot r}}{b_{o}+h_{o} \cdot h_{1}}
$$

Onde $\Delta b$ é a variação absoluta em altura, $\Delta h$ é a variação da altura e $h_{0}$ é a altura antes da entrada nos cilindros, $\mathrm{h}_{1}$ é altura após a saída, e bo é a largura antes da entrada nos cilindros.

\subsubsection{Modelo de Köster}

Köster apresentou a seguinte fórmula para o cálculo do alargamento:

$$
\frac{\varphi_{b}}{\varphi_{h}}=-e^{\left(-c_{b p} \frac{b_{o}}{l d}\right)}
$$

Onde a correlação da formula de Köster para temperatura, $\mathrm{Cb}_{\mu}$, pode ser calculado (14):

$$
C_{b \mu}=\frac{T_{r e f}}{T_{\text {lam }}}
$$

Sendo que $b_{0}$ corresponde a largura antes da entrada nos cilindros, Id corresponde o arco de contato, a $T_{\text {ref }}$ é a temperatura de referência e $T_{\text {lam }}$ é a temperatura de laminação.

\subsection{Cálculo da Força de Laminação}

A força é calculada em função da tensão que atua sobre um elemento de área (15):

$$
F=A_{d} \cdot k_{w} \text { (15) }
$$

Onde $A_{d}$ é a área de contato entre a barra e o cilindro e $k_{w}$ é a resistência a deformação. $\mathrm{k}_{\mathrm{w}}$ é determinada a partir da equação (16):

$$
\mathrm{k}_{\mathrm{w}}=\mathrm{k} \cdot \mathrm{k}_{\mathrm{f}} \text { (16) }
$$

Sendo $k_{f}$ a tensão de escoamento calculada por (17):

$$
\mathrm{K}_{\mathrm{f}}=\sigma \cdot(1+0,05 \cdot \dot{\varphi})(17)
$$

Onde $\sigma$ é a tensão de escoamento e é a velocidade de deformação.

\section{MATERIAIS E MÉTODOS}

A partir das barras de aço AISI 1020 foram confeccionados os corpos de prova nos formatos e dimensões apresentados na Figura 4. 


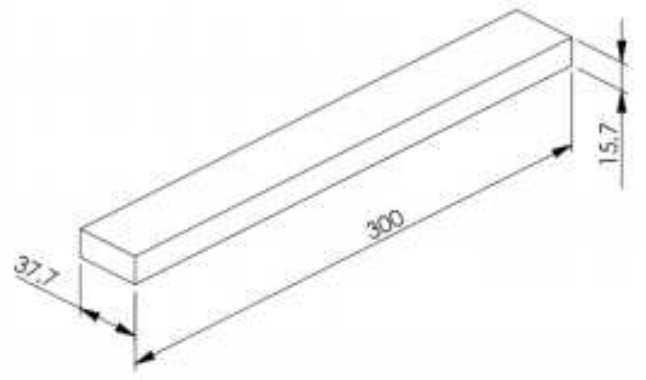

Figura 4 - Representação das dimensões iniciais da barra de aço AISI 1020.

A composição do aço AISI 1020 se encontra na Tabela 1.

Tabela 1 - Composição química do aço AISI 1020.

\begin{tabular}{|c|c|c|c|c|}
\hline AISI & C & Si & P & S \\
\hline 1020 & 0,21 & 0,42 & 0,01 & 0,02 \\
\hline
\end{tabular}

Foram confeccionados 6 corpos de prova onde foram verificadas as dimensões finais após 1 passe de laminação, sem adição de lubrificantes.

Os testes experimentais de laminação foram realizados em um laminador tipo Duo, GarbeLahmayer\&Co AG, com diâmetro dos cilindros lisos de 178,8mm, a uma temperatura de laminação de $1000^{\circ} \mathrm{C}$ e luz regulada com o auxílio de um relógio comparador para reduções de $15 \%$ e $20 \%$ em altura, assim, buscou-se alturas finais de $13,34 \mathrm{~mm}$ e $12,56 \mathrm{~mm}$, respectivamente.

Os corpos de prova foram aferidos com auxílio de paquímetro, antes e após a laminação para verificação do alargamento, comprimento e altura alterados.

\section{RESULTADOS E DISCUSSÃO}

A partir da fórmula de Ekelund (equação 10), obteve-se o valor do coeficiente de atrito $(\mu)$ de 0,55 . Também foi encontrado o valor do ângulo de agarre $(\alpha)$ para as duas reduções, tanto para $9,04^{\circ}$ quanto para $11,24^{\circ} \mathrm{e}$, com isso, foi possível determinar a condição de agarre $(\mu>\operatorname{tg} \alpha)$.

$\mathrm{Na}$ Tabela 2 estão apresentados os valores calculados a partir dos modelos matemáticos de Siebel, Tafel e Sedlazczek, e Köster para o alargamento com reduções aproximadas de $15 \%$ e $20 \%$ em relação à altura da barra laminada e os valores obtidos experimentalmente.

Tabela 2 - Larguras obtidas a partir dos modelos teórico e experimental.

\begin{tabular}{|c|c|c|c|c|c|c|c|c|c|}
\cline { 2 - 10 } \multicolumn{1}{c|}{} & \multicolumn{9}{c|}{ Alargamento } \\
\cline { 2 - 10 } \multicolumn{1}{c|}{} & \multicolumn{2}{c|}{ Siebel } & \multicolumn{2}{c|}{ Tafel e Sedlaczek } & \multicolumn{2}{c|}{ Köster } & \multicolumn{2}{c|}{ Experimental } \\
\cline { 2 - 10 } \multicolumn{1}{c|}{} & Inicial & $15 \%$ & $20 \%$ & $15 \%$ & $20 \%$ & $15 \%$ & $20 \%$ & $15 \%$ & $20 \%$ \\
\hline b (mm) & 37,7 & 38,39 & 39,02 & 38,98 & 39,71 & 38,40 & 39,42 & 38,4 & 38,8 \\
\hline
\end{tabular}

Os valores estão representados em gráfico de barras para melhor visualização comparativa dos resultados teóricos com os experimentais conforme exibido na Figura 5. 


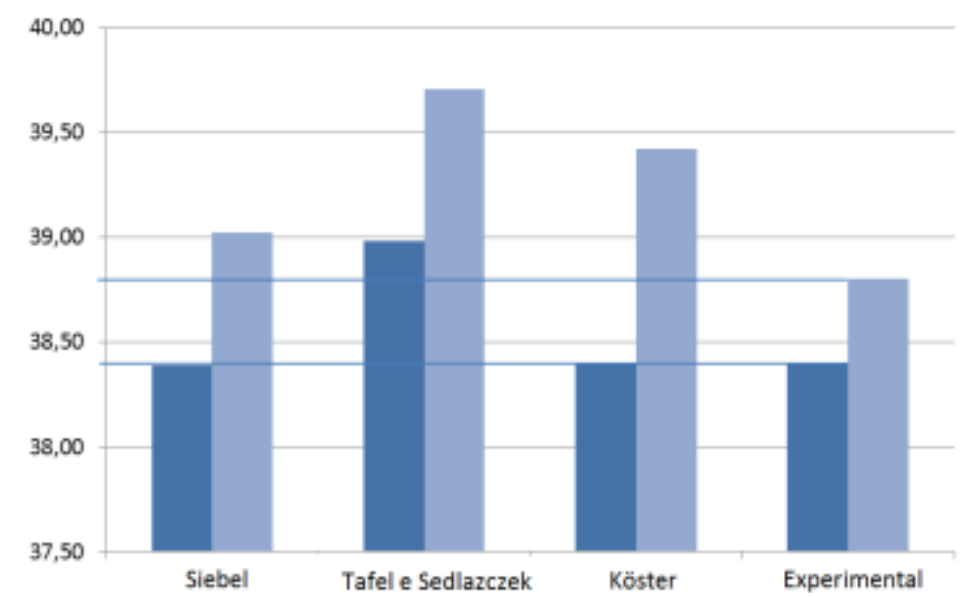

Figura 5 - Representação gráfica dos resultados para alargamento.

As barras laminadas estão apresentadas na Figura 6, onde se visualiza a lei de constância de volume, pois onde há maior redução em altura houve maior alargamento e alongamento.

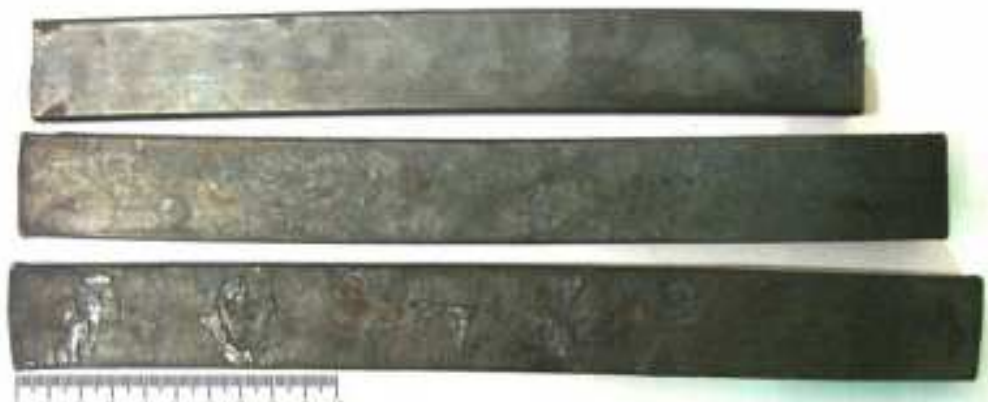

Figura 6 - Barras antes da laminação, laminada com redução aproximada de 15\% e laminada com redução aproximada de $20 \%$.

Os dados relacionados à determinação da força e de tensões no processo foram calculados a partir de modelos teóricos e estão apresentados na Tabela 3.

Tabela 3 - Variáveis obtidas a partir de modelos teóricos.

\begin{tabular}{|l|c|c|}
\cline { 2 - 3 } \multicolumn{1}{c|}{} & Redução Aprox. 15\% & Redução Aprox. 20\% \\
\hline Ângulo de agarre $(\alpha)$ & $9^{\circ}$ & $11,2^{\circ}$ \\
\hline Arco de contato $\left(\mathrm{l}_{\mathrm{d}}\right)$ & $14,02 \mathrm{~mm}$ & $17,43 \mathrm{~mm}$ \\
\hline Área de Contato $\left(\mathrm{A}_{\mathrm{d}}\right)$ & $533,46 \mathrm{~mm}^{2}$ & $666,69 \mathrm{~mm}^{2}$ \\
\hline Coeficiente de Plasticidade $(n)$ & 0,4 & 0,4 \\
\hline Força de Laminação $(\mathrm{F})$ & $57 \mathrm{KN}$ & $76 \mathrm{KN}$ \\
\hline Largura média $\left(\mathrm{b}_{\mathrm{m}}\right)$ & $38,05 \mathrm{~mm}$ & 38,25 \\
\hline Resistência à deformação $\left(\mathrm{k}_{\mathrm{w}}\right)$ & $108 \mathrm{~N} / \mathrm{mm}^{2}$ & $114 \mathrm{~N} / \mathrm{mm}^{2}$ \\
\hline Tensão de Escoamento $\left(\mathrm{k}_{\mathrm{f}}\right)$ & $82 \mathrm{~N} / \mathrm{mm}^{2}$ & $82 \mathrm{~N} / \mathrm{mm}^{2}$ \\
\hline
\end{tabular}

As barras estudadas foram analisadas metalograficamente para avaliar sua modificação microestrutural. A Figura 7 mostra a metalografia da barra comercial antes do estudo de laminação executado. 


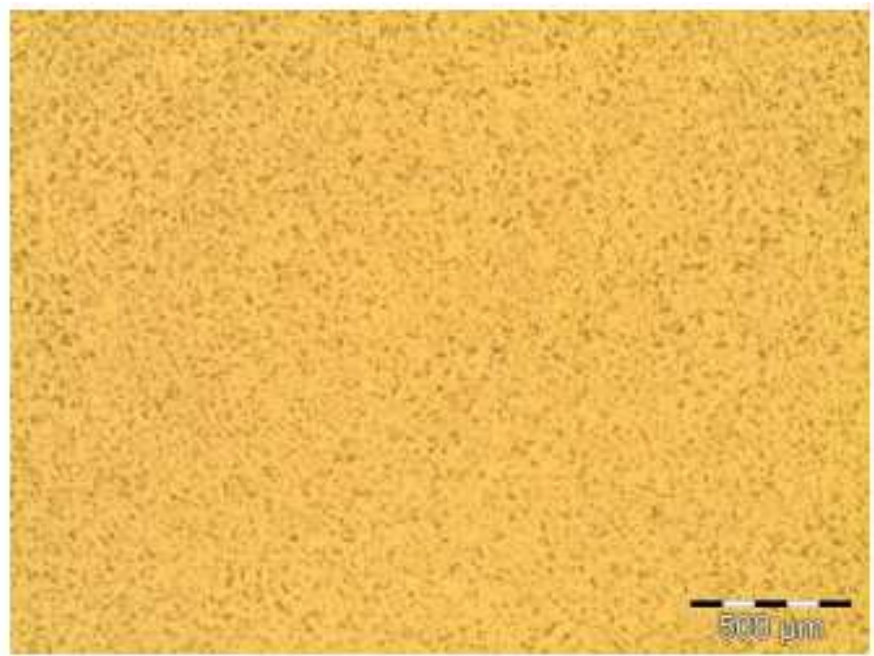

Figura 7 - Microestrutura inicial da barra de aço AISI 1020 (50x).

A metalografia mostra uma microestrutura homogênea típica do aço AISI 1020 com pouco percentual de perlita dentro de uma matriz de ferrita devido ao baixo teor de carbono da composição. A Figura 8 exibe a microestrutura da barra inicial com magnificação de 500x.

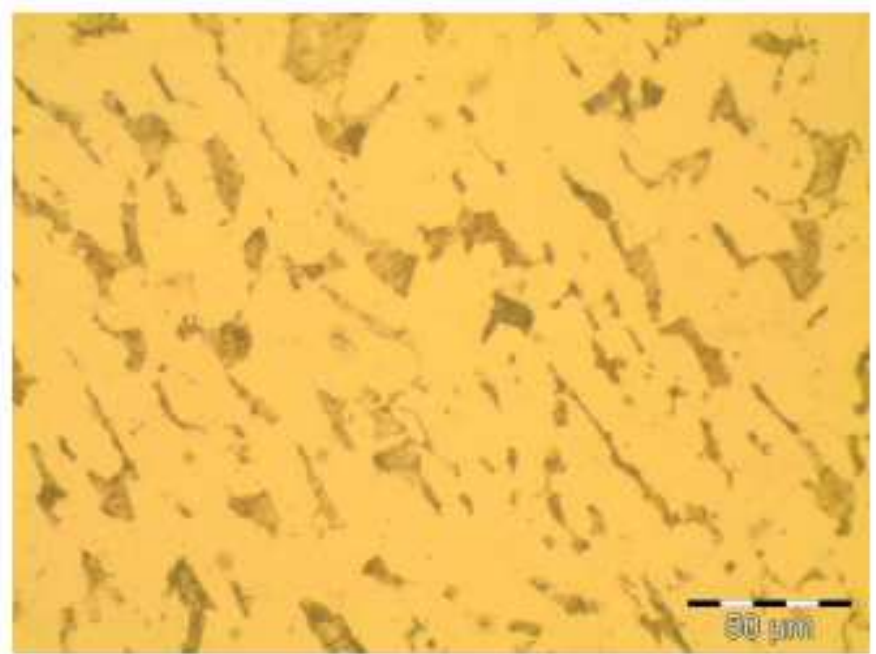

Figura 8 - Microestrutura inicial da barra de aço AISI 1020 (500x).

Pode-se observar o tamanho de grão médio de $50 \mu \mathrm{m}$ de ferrita e um grão refinado de perlita entre 20 e $30 \mu \mathrm{m}$ com um leve alinhamento em diagonal da anisotropia proveniente do processo de conformação anterior. A Figura 9 apresenta a microestrutura da barra laminada com redução de $15 \%$ na altura com Ângulo de agarre de $9^{\circ}$ e magnificação de 500 vezes. 


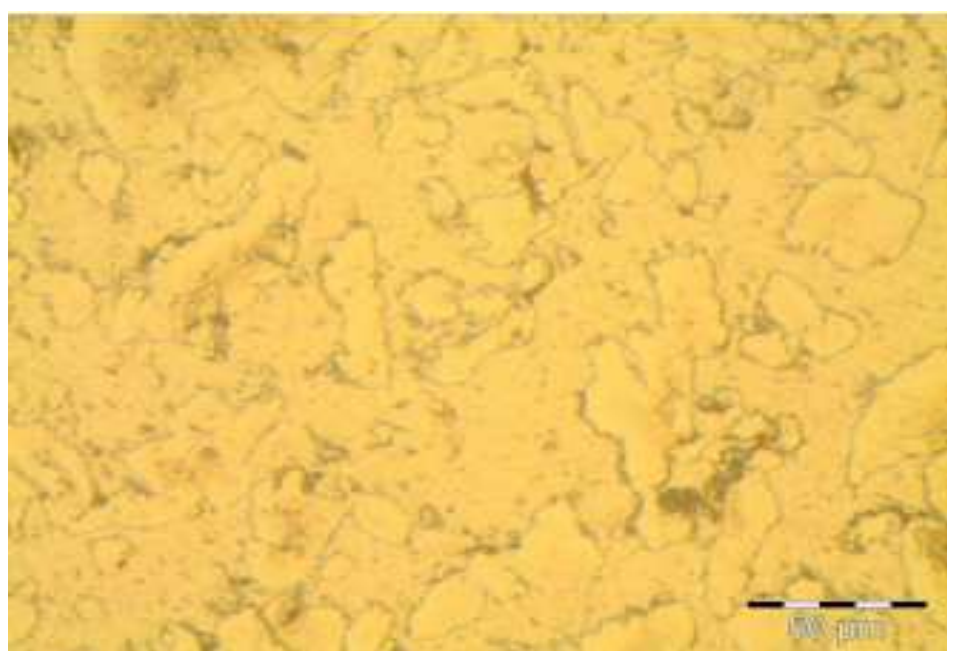

Figura 9 - Microestrutura da barra de aço AISI 1020 com 15\% de redução (500x).

Nota-se a diminuição do tamanho de grão entre 10 e $20 \mu \mathrm{m}$ da ferrita e não ficou evidente a orientação da laminação juntamente com a difusão de diversos grãos. A Figura 10 exibe a microestrutura da barra laminada com $20 \%$ de redução da altura.

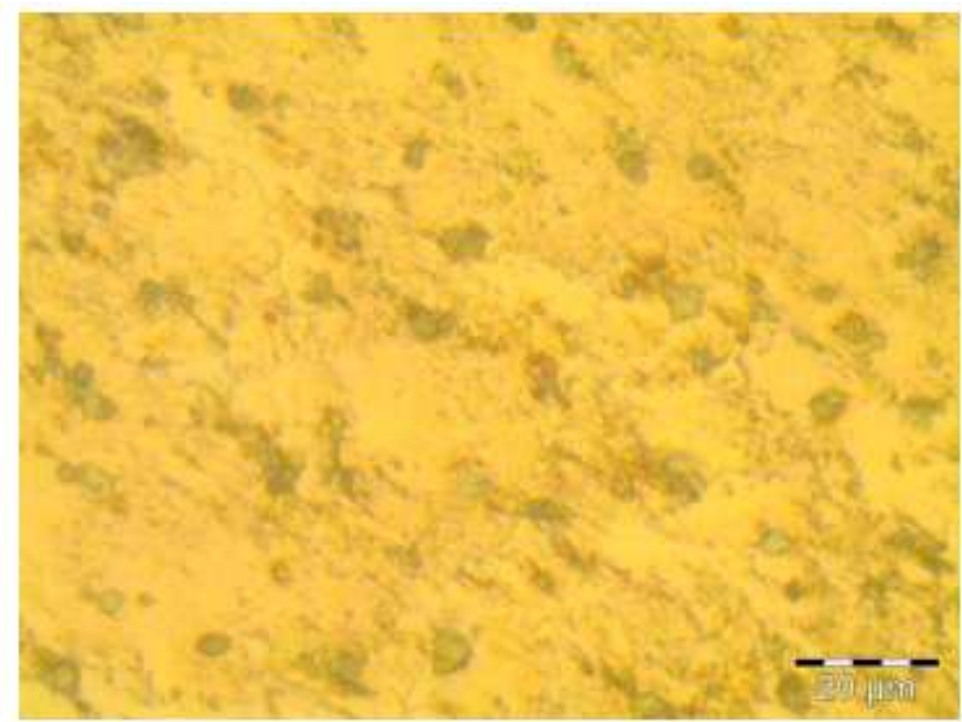

Figura 10 - Microestrutura da barra de aço AISI 1020 com 20\% de redução (1000x).

Na Figura 10 evidencia-se que a microestrutura tem uma leve orientação e os grãos médios da Perlita ficaram entre 5 e menor que $10 \mu \mathrm{m}$ totalmente dispersos com uma distribuição homogênea sobre a matriz ferrítica com tamanho de grão entre 10 e 20 $\mu \mathrm{m}$.

\section{CONCLUSÃO}

O modelo matemático que mais se aproximou dos resultados experimentais foi o modelo de Siebel, considerando as duas reduções (redução de 15\% e 20\%), porém, o modelo de Köster foi o mais preciso na redução aproximada de $15 \%$. O modelo de Tafel e Sedlazczek apresentou valores mais divergentes comparados com os resultados experimentais, podendo ser descartado para calcular a redução de uma barra laminada. Conforme o percentual de redução é aumentado, os resultados teóricos se distanciam mais dos experimentais, podendo ser originado em função da 
dificuldade de obtenção dos dados para equação. Observa-se que as microestruturas das barras laminadas com redução de 15 e 20\% na altura refinaramse, quando comparadas com a microestrutura da barra inicial.

\section{Agradecimentos}

Os autores agradecem ao Conselho Nacional de Desenvolvimento Científico e Tecnológico (CNPq) e à Coordenação de Aperfeiçoamento de Pessoal de Nível Superior (CAPES) pelo financiamento das bolsas de estudo, ao Laboratório de Transformação Mecânica (LdTM) e à Universidade Federal do Rio Grande do Sul (UFRGS).

\section{REFERÊNCIAS}

1 SCHAEFFER, L. Conformação Mecânica. 2. ed. Porto Alegre: Imprensa Livre, 2004.

2 MILANEZ, A. Estudo da Calibração de barras chatas laminadas a quente em um laminador Trio. Dissertação de Mestrado. Universidade Federal do Rio Grande do Sul, 2006.

3 Processo de laminação. Disponível em: < http://mmborges.com/processos/Conformacao/cont_html/laminacao.htm>. Acesso em: 17/05/2013.

4 MORAES, A. S. Estudo teórico-experimental da calibração de barras redondas laminadas a quente. Dissertação de Mestrado. Universidade Federal do Rio Grande do Sul, 2010. 